\title{
Bounds for 2-D angle-of-arrival estimation with separate and joint processing
}

\author{
Laurence Mailaender
}

\begin{abstract}
Cramer-Rao bounds for one- and two-dimensional angle-of-arrival estimation are reviewed for generalized 3-D array geometries. Assuming an elevated sensor array is used to locate sources on a ground plane, we give a simple procedure for drawing $x$-y location confidence ellipses from the Cramer-Rao covariance matrix. We modify the ordinary bounds for the case of "separate" 1-D estimates and numerically compare this with the full, joint bound. We prove that "separate" processing is optimal for a Uniform Cross Array with a single source, and that it is not optimal for the L-shaped array. A trade-off emerges between location accuracy and array height: for distant sources, increased height generally reduces error. When more than one source is present, significant gains are obtained from joint processing. We also show useful gains for distant sources by adding out-of-plane sensors in an " $L+Z$ " configuration with joint processing. These comparisons can aid system designers in deciding between separate and joint processing approaches.
\end{abstract}

\section{Introduction}

Transmitting sources may be located by estimating the angles-of-arrival at a receiving array if the "direct path" is present, i.e., the straight line path between source and destination. Angle-of-arrival (AOA) estimation may be efficiently performed by well-known approaches such as MUSIC and ESPRIT [1], and the performance of these algorithms has been shown to approach the Cramer-Rao lower bound (CRLB) at moderate SNR [2]. The CRLB is well-studied for one-dimensional (1-D) angle estimation, and 1-D bounds have been derived under various assumptions about the source signals, e.g., the Conditional Model Assumption (CMA) [2,3] and the Unconditional Model Assumption (UMA) [4,5]. Yet many realworld applications require a two-dimensional (2-D) AOA estimation (e.g., azimuth and elevation) because the sources and receive array may not lie in a single plane.

2-D AOA estimation may be computationally complex, and several authors have proposed sensor geometries which allow the 2-D problem to be broken into multiple 1-D problems. In [6], the "L-shaped" array (LSA) and "two L-shaped" array (2LSA) are used with the Propagator Method to avoid complexity associated

Correspondence: Im@lgsinnovations.com LGS Innovations LLC, Florham Park, NJ, USA with "pair matching." In [7], algorithms using the LSA are discussed, and the notion of separate 1-D processing of each linear sub-array in elevation-elevation coordinates is presented. Two 1-D searches may be made using parallel uniform arrays [8]. The 2LSA is used with separate processing in each of three axes in [9].

These works motivate us to consider the 2-D CRLB with joint and separate processing. The 2-D CRLB can be found in the literature under both CMA [10,11] and UMA [12]. However, little attention has been paid to the CRLB for "separate" processing. The 2-D CRLB can also be used as a tool to optimize antenna array geometry [13].

In this article, we assume that source location is performed by 2-D AOA estimation followed by trigonometric projection to the ground plane. To keep the approach simple, we assume the Earth's surface is a plane; in principle, topographic maps could also be incorporated. In a typical implementation, a single array at known height is used to locate the sources. Using the classic "change of variables" approach, the CRLB for 2$\mathrm{D}$ AOA is converted to the CRLB for $(x, y)$ position. We review the procedure for drawing 2-D error ellipses corresponding to any desired confidence level (e.g., containing a 95\% of all erroneous positions) for estimators that achieve this CRLB. Next, we show how the existing 2-D AOA CRLBs may be modified from the optimal "joint"

\section{SpringerOpen ${ }^{\circ}$}

(C) 2011 Mailaender; licensee Springer. This is an Open Access article distributed under the terms of the Creative Commons Attribution License (http://creativecommons.org/licenses/by/2.0), which permits unrestricted use, distribution, and reproduction in any medium, provided the original work is properly cited. 
bound into (generally suboptimal) "separate" bounds to handle lower complexity 1-D searches. We develop the 2-D CRLB under two coordinate systems: azimuth-elevation (for joint processing) and elevation-elevation (for separate processing). In addition, some basic facts about the "separability" of the CRLB are proved for simple sensor configurations, and the 2-D bound is proved to be non-increasing in the number of sensors.

The remainder of the article is organized as follows. In Sect. 2, we review the definitions of the general array response vectors under different $2-\mathrm{D}$ coordinate systems, and give a procedure for drawing error ellipses corresponding to the CRLB error covariance matrix. In Sect. 3, we review results from the literature on 1-D and 2-D CRLBs, compute the bounds for both coordinate

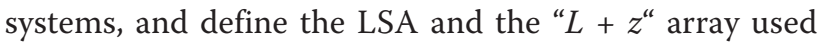
later in this article. In Sect. 4, we show how to modify the 2-D CRLB for "separate" angle processing, and we give some proofs regarding the separability and monotonicity of the CRLB. Section 5 contains our numerical results, where separate and joint processing is compared, with 1 or more sources present. Section 6 contains a summary and our conclusions.

Regarding notation, vectors will appear as a, matrices as $\mathbf{A}$, and the Hermitian-transpose as $\mathbf{A}^{\mathrm{H}}$. The "all 1's" column vector of length $K$ is denoted $1_{k}$. The notation $\mathbf{A}_{\odot} \mathbf{B}$ indicates the Schur-Hadamard element-by-element multiplication of same-sized objects, the notation $\mathbf{A}_{\otimes} \mathbf{B}$ denotes a Kronecker product.

\section{Geometrical models}

\section{a. Coordinate systems}

Figure 1 shows an $(x, y, z)$ coordinate axis, with the signal direction of arrival defined in both azimuth-elevation ("az-el"), $(\theta, \phi)$, and elevation-elevation ("el-el"), $(\alpha, \beta)$ coordinates. As noted in [7], the el-el system allows two "separate" 1-D angle of arrival measurements from two Uniform Linear Arrays to be combined. Hence the el-el format potentially allows an algorithmic complexity reduction by solving separate $1-\mathrm{D}$ problems.

The array response for the $n$th element in a general array is most simply written in el-el form,

$$
a_{n}(\alpha, \beta, \gamma)=\exp \left(-j \frac{2 \pi}{\lambda}\left(x_{n} \cos (\alpha)+y_{n} \cos (\beta)+z_{n} \cos (\gamma)\right)\right)
$$

where $x_{n}, y_{n}, z_{n}$ define the element position (in meters) relative to the array axes, $\alpha, \beta, \gamma$ are the elevation angles relative to the three axes, and $\lambda$ is the signal's wavelength in meters/cycle. This expression has an intuitively pleasing symmetry: nature should not care which axis is labeled as $x$, etc. Only two angles are needed to determine the direction of arrival, so we can define the third elevation angle from the other two, or, $\gamma=\operatorname{acos}\left(\sqrt{1-\cos ^{2}(\alpha)-\cos ^{2}(\beta)}\right)$ where acos $(x)$ denotes the inverse cosine. Inserting into (2.1) gives the general array response in el-el coordinates,

$$
a_{n}(\alpha, \beta)=\exp \left(-j \frac{2 \pi}{\lambda}\left(x_{n} \cos (\alpha)+\gamma_{n} \cos (\beta)+z_{n}\left(1-\cos ^{2}(\alpha)-\cos ^{2}(\beta)\right)^{1 / 2}\right)\right)
$$

We will consider el-el measurements to be inconsistent (meaning no valid direction of arrival can be found) if $1<\cos ^{2}(\alpha)+\cos ^{2}(\beta)$. For consistent angles, we may transform el-el to az-el by noting that:

$$
\begin{aligned}
& \gamma=\varphi \\
& \cos (\alpha)=\sin (\gamma) \cos (\theta) \\
& \cos (\beta)=\sin (\gamma) \sin (\theta)
\end{aligned}
$$

Direct substitution leads to the general array response in az-el form [2],

$$
a_{n}(\theta, \varphi)=\exp \left(-j \frac{2 \pi}{\lambda}\left(x_{n} \sin (\varphi) \cos (\theta)+y_{n} \sin (\varphi) \sin (\theta)+z_{n} \cos (\varphi)\right)\right)(2.4)
$$

Note that the az-el system has no possibility of inconsistency, but for the special cases of $\phi=\{0, \pi\}$ the angle $\theta$ cannot be estimated (hence, the 2-D CRLB does not exist). Equations 2.2 and 2.4 will be used to create array

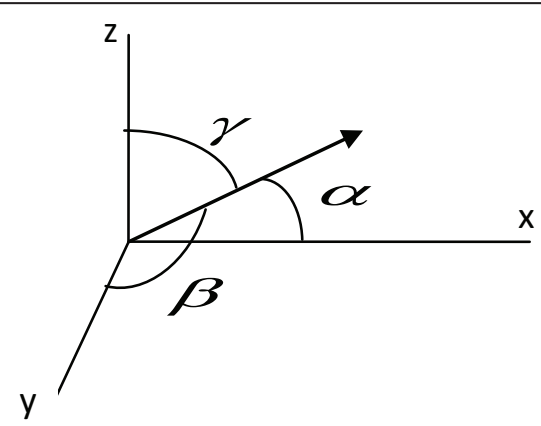

Elevation-Elevation Coordinates



Azimuth-Elevation Coordinates

Figure 1 Coordinate axes. 
response vectors for 2-D and 3-D array geometries in following sections. Writing array element coordinates as vectors $\mathbf{p}_{x}, \mathbf{p}_{y}, \mathbf{p}_{z}$, the array response vector from the $m$ th source is written $\mathbf{a}\left(\theta_{m}, \phi_{m}\right)$ or $\mathbf{a}\left(\alpha_{m}, \beta_{m}\right)$

Angle estimates are mapped to $(x, y)$ position estimates via either,

$$
\left[\begin{array}{l}
x \\
y
\end{array}\right]=F_{\mathrm{e}}\left(\left[\begin{array}{l}
\alpha \\
\beta
\end{array}\right]\right)=\left[\begin{array}{l}
z \cos (\alpha)\left(1-\cos ^{2}(\alpha)-\cos ^{2}(\beta)\right)^{-1 / 2} \\
z \cos (\beta)\left(1-\cos ^{2}(\alpha)-\cos ^{2}(\beta)\right)^{-1 / 2}
\end{array}\right]
$$

or,

$$
\left[\begin{array}{l}
x \\
y
\end{array}\right]=F_{\mathrm{a}}\left(\left[\begin{array}{l}
\theta \\
\varphi
\end{array}\right]\right)=\left[\begin{array}{l}
z \cos (\theta) \tan (\varphi) \\
z \sin (\theta) \tan (\varphi)
\end{array}\right]
$$

\section{b. Location error ellipses}

In a location system, the sources' $(x, y)$ coordinates are of ultimate interest, however, we assume the AOA is estimated first, so the system designers can take advantage of modern angle estimation techniques (e.g., MUSIC, ESPRIT, etc.). The estimated angles are then converted to $(x, y)$ estimates, which have a different CRLB. In this section, we show how to draw an error ellipse in the $(x$, $y$ ) plane, containing an area with a specified probability level, corresponding to an $(x, y)$ estimator that achieves the CRLB.

For positive-definite matrix, $\mathbf{A}$, the equation $\mathbf{x}^{\mathrm{T}} \mathbf{A x}=$ 1 defines an ellipse where the length of each axis from the origin is the inverse square-root of the eigenvalues of $\mathbf{A}[14]$. Let $\mathbf{C}=E\left\{(\boldsymbol{\theta}-\hat{\boldsymbol{\theta}})(\boldsymbol{\theta}-\hat{\boldsymbol{\theta}})^{\mathrm{H}}\right\} \triangleq \mathbf{J}^{-1}$ be the positive-definite error covariance matrix for an estimator that achieves the CRLB. This covariance matrix defines the error "concentration ellipse" with the smallest possible volume (or area, in the 2-D case) [15]. Consider the equation,

$$
\begin{aligned}
c^{-2} \boldsymbol{\theta}^{\mathrm{T}} \mathbf{C}^{-1} \boldsymbol{\theta} & =1 \\
c^{-2} \boldsymbol{\theta}^{\mathrm{T}} \mathbf{V} \mathbf{D}^{-1} \mathbf{V}^{\mathrm{H}} \boldsymbol{\theta} & =1
\end{aligned}
$$

where $\mathrm{V}, \mathrm{D}$ contain the eigenvectors and eigenvalues of $\mathbf{C}$. Note (2.7) has solutions $\boldsymbol{\theta}=c \lambda_{i}^{1 / 2} \mathbf{v}_{i}, i=1,2$ corresponding to the major and minor axes. The values $\lambda_{i}^{1 / 2}$ are the error standard deviations on the orthogonal axes, and constant " $c$ " scales the axis lengths proportionally. We want to scale the ellipse so that it contains some fraction of the total probability, referred to as a "confidence factor" (CF). As shown in [16], the desired scaling is,

$$
c=\sqrt{-2 \ln (1-\mathrm{CF})}
$$

For example, we can draw an ellipse containing 95\% of all estimates, by setting $c=2.448$.

Note that each point on the error ellipse must satisfy $c^{2}=(\Delta x)^{2} / \lambda_{1}+(\Delta y)^{2} / \lambda_{2}$, hence we can determine the points on the ellipse (2.7) by stepping through values $\Delta x$ $=0$ through $c \lambda_{i}^{1 / 2}$ along one axis, and solving for $\Delta y=$ $\left(\left(c^{2}-(\Delta x)^{2} / \lambda_{1}\right) \lambda_{2}\right)^{1 / 2}$. This creates one quadrant of the ellipse; the rest is produced by symmetry. Finally, the ellipse is centered at the true position, $\left(\theta_{1}, \theta_{2}\right)$.

\section{Review of CRLB}

\section{a. General principles}

The CRLB $[2,15,16]$ gives the lower limit on estimation error among all unbiased estimators. The bound is a useful performance metric, as many practical algorithms have been found to closely approach it at reasonable SNR values [2]. Moreover, the CRLB is a flexible tool that allows modeling many kinds of estimation problems. Most problems involve so-called nuisance variables in addition to the variables of direct interest. When deriving the bound, nuisance parameters that are known are treated as constants, while those that are unknown must be included in the parameter vector, $\boldsymbol{\theta}$. The Fisher matrix is given by

$$
\mathbf{J}(\boldsymbol{\theta})=E\left\{\left(\frac{\mathrm{d}}{\mathrm{d} \boldsymbol{\theta}} \Lambda(\boldsymbol{\theta})\right)\left(\frac{\mathrm{d}}{\mathrm{d} \boldsymbol{\theta}} \Lambda(\boldsymbol{\theta})\right)^{\mathrm{H}}\right\}
$$

where $\Lambda(\boldsymbol{\theta})$ denotes the log-likelihood of the received vector given the parameters $\boldsymbol{\theta}$, and the expectation is over the received signal conditioned on $\boldsymbol{\theta}$. The inverse of the Fisher matrix bounds the achievable error covariance, $\mathbf{C} \geq \mathbf{J}^{-1}$ and the diagonal elements of $\mathbf{J}^{-1}$ bound the estimation error of the individual variables.

If a new parameter vector is related to the old one via a change of variables, $\mathbf{w}=F(\boldsymbol{\theta})$, then [15],

$$
\mathbf{J}^{-1}(\mathbf{w})=\dot{\mathbf{F}}^{T} \mathbf{J}^{-1}(\boldsymbol{\theta}) \dot{\mathbf{F}}, \quad(\dot{\mathbf{F}})_{i, j}=\frac{\mathrm{d} w_{j}}{\mathrm{~d} \theta_{i}}
$$

gives the new CRLB.

\section{b. Bounds for $A O A$}

Assume a system with $N$ sensors, $M$ sources, and $K$ data snapshots. Initially, consider the sources to lie in the plane of the array (1-D angle estimation). The signal vector received at the array for the $k$ th snapshot is,

$$
\mathbf{r}(k)=\mathbf{A}\left(\boldsymbol{\theta}_{1}\right) \mathbf{s}(k)+\mathbf{n}(k)
$$

with $\mathbf{r} \in C^{N \times 1}$, array response matrix $\mathbf{A} \in C^{N \times M}$, source vector $\mathbf{s} \in C^{M \mathrm{x} 1}$, and where noise vector $\mathbf{n} \in$ $C^{N \times 1}$ is assumed to be proper, complex Gaussian with 
covariance $\sigma_{n}^{2} \mathbf{I}_{N}$. Different CRLBs can be derived depending on assumptions regarding the transmitted signals. As shown in [2], three assumptions about the transmitted signals $\mathbf{s}(k)$ are commonly used: $\mathbf{s}(k)$ is considered unknown and deterministic (CMA, or "deterministic" bound); $\mathbf{s}(k)$ is considered a sample of a complex Gaussian noise process of unknown covariance (UMA, or "stochastic" bound); or finally $\mathbf{s}(k)$ can be considered known (most optimistic bound). It seems unlikely that the signal being located would be completely known, hence we will consider only the deterministic and stochastic bounds (exceptions are cooperative transmitters acting as beacons, or transmitters using standardized waveforms having certain features that are known $a$ priori).

Under 1-D, deterministic assumptions, the unknown parameter vector is $\boldsymbol{\theta} \triangleq\left[\theta_{1}, \ldots, \theta_{M}, \operatorname{Re}\{\mathbf{s}(1)\}^{\mathrm{T}}, \operatorname{Im}\{\mathbf{s}(1)\}^{\mathrm{T}}, \ldots, \operatorname{Re}\{\mathbf{s}(K)\}^{\mathrm{T}}, \operatorname{Im}\{\mathbf{s}(K)\}^{\mathrm{T}}\right]^{\mathrm{T}}$ consisting of $M+2 M K$ real parameters. The $M \times M$ upper-left corner of the inverse of the Fisher matrix [2-4] corresponding to the parameters of direct interest (dropping the $\mathbf{A}(\boldsymbol{\theta})$ notation for convenience) is,

$$
\mathbf{J}_{\mathrm{d}, 1}^{-1}=\frac{\sigma_{n}^{2}}{2 K}\left(\operatorname{Re}\left\{\mathbf{D}^{\mathrm{H}}\left(\mathbf{I}-\mathbf{A}\left(\mathbf{A}^{\mathrm{H}} \mathbf{A}\right)^{-1} \mathbf{A}^{\mathrm{H}}\right) \mathbf{D} \odot \mathbf{P}^{\mathrm{T}}\right\}\right)^{-1} .
$$

Here,

$$
\begin{aligned}
& \mathbf{A}(\theta)=\left[\mathbf{a}\left(\theta_{1}\right), \ldots, \mathbf{a}\left(\theta_{M}\right)\right] \\
& \mathbf{D}(\theta)=\left[\frac{\mathrm{d}}{\mathrm{d} \theta_{1}} \mathbf{a}\left(\theta_{1}\right), \ldots, \frac{\mathrm{d}}{\mathrm{d} \theta_{M}} \mathbf{a}\left(\theta_{M}\right)\right] \\
& \mathbf{P}=E\left\{\mathbf{s} \mathbf{s}^{\mathrm{H}}\right\}
\end{aligned}
$$

The subscript "d,1" indicates this is a deterministic, 1D bound. Under 1-D stochastic assumptions, the unknown parameter vector is $\boldsymbol{\theta} \triangleq\left[\theta_{1}, \ldots, \theta_{M}, \operatorname{Re}\left\{R_{1,1}\right\}, \ldots, \operatorname{Re}\left\{R_{N, N}\right\}, \operatorname{Re}\left\{R_{1,2}\right\} \operatorname{Im}\left\{R_{1,2}\right\}, \ldots, \operatorname{Im}\left\{R_{N, N-1}\right\}\right]^{\mathrm{T}}$ consisting of $M+N^{2}$ unknowns (accounting for Hermitian symmetry), where $R_{i, j}$ is the $i, j$ th element of the signal covariance matrix. The $M \times M$ upper-left corner of the inverse of the Fisher matrix $[2,4,5]$ corresponding to the parameters of direct interest is,

$$
\begin{aligned}
\mathbf{J}_{s, 1}^{-1}= & \frac{\sigma_{n}^{2}}{2 K}\left(\operatorname{Re}\left\{\mathbf{D}^{\mathrm{H}}\left(\mathbf{I}-\mathbf{A}\left(\mathbf{A}^{\mathrm{H}} \mathbf{A}\right)^{-1} \mathbf{A}^{\mathrm{H}}\right) \mathbf{D} \odot\left(\mathbf{P A}^{\mathrm{H}} \mathbf{R}^{-1} \mathbf{A} \mathbf{P}^{\mathrm{T}}\right)\right\}\right)^{-1} \\
\mathbf{R} & =\mathbf{A P A}^{\mathrm{H}}+\sigma_{n}^{2} \mathbf{I}
\end{aligned}
$$

where the subscript "s,1" denotes a 1-D stochastic bound. The matrices A, D, $\mathbf{P}$ are as in (3.5). The thermal noise variance may also be considered an "unknown" but the bounds (3.4), (3.6) remain unchanged [2].

Next consider a 2-D signal model where it is understood that $\left(\boldsymbol{\theta}_{1}, \boldsymbol{\theta}_{2}\right)$ may correspond to either $\left(\alpha_{n}, \beta_{n}\right)$, or $\left(\theta_{n}, \phi_{n}\right)$ for all $n$ users,

$$
\mathbf{r}(k)=\mathbf{A}\left(\boldsymbol{\theta}_{1}, \boldsymbol{\theta}_{2}\right) \mathbf{s}(k)+\mathbf{n}(k)
$$

At first, it may appear that the new unknowns can simply be appended to the $\boldsymbol{\theta}$ vector and the previous formulas used. However, the matrix multiplication with the $\odot$ term in (3.4), (3.6) will no longer be dimensionally correct, so a new formula is needed. Deriving compact expressions for these formulas turns out to be extremely difficult; however, all the needed results are available in the literature. Under 2-D deterministic assumptions, the unknown parameter vector is $\boldsymbol{\theta} \triangleq\left[\theta_{1,1}, \ldots, \theta_{1, M}, \theta_{2,1}, \ldots \theta_{2, M}, \operatorname{Re}\{\mathbf{s}(1)\}^{\mathrm{T}}, \operatorname{Im}\{\mathbf{s}(1)\}^{\mathrm{T}}, \ldots, \operatorname{Re}\{\mathbf{s}(K)\}^{\mathrm{T}}, \operatorname{Im}\{\mathbf{s}(K)\}^{\mathrm{T}}\right]^{\mathrm{T}}$ consisting of $2 M+2 M K$ real parameters. The $2 M \times 2 M$ upper-left corner of the inverse of the Fisher matrix $[10,11]$ corresponding to the parameters of direct interest is, ${ }^{\text {a }}$

$$
\begin{aligned}
\mathrm{I}_{\mathrm{d}, 2}^{-1}=\frac{\sigma_{n}^{2}}{2 K}\left(\operatorname{Re}\left\{\mathbf{D}^{\mathrm{H}}\left(\mathbf{I}-\mathbf{A}\left(\mathrm{A}^{\mathrm{H}} \mathbf{A}\right)^{-1} \mathbf{A}^{\mathrm{H}}\right) \mathbf{D} \odot\left(\mathbf{1}_{2} \mathbf{1}_{2}^{\mathrm{T}} \otimes \mathbf{P}^{\mathrm{T}}\right)\right\}\right)^{-1} \\
\mathbf{D}(\boldsymbol{\theta})=\left[\frac{\mathrm{d}}{\mathrm{d} \theta_{1,1}} \mathbf{a}\left(\theta_{1,1}, \theta_{2,1}\right), \ldots, \frac{\mathrm{d}}{\mathrm{d} \theta_{1, M}} \mathbf{a}\left(\theta_{1, M}, \theta_{2, M}\right), \frac{\mathrm{d}}{\mathrm{d} \theta_{2,1}} \mathbf{a}\left(\theta_{1,1}, \theta_{2,1}\right), \ldots, \frac{\mathrm{d}}{\mathrm{d} \theta_{2, M},} \mathbf{a}\left(\theta_{1, M}, \theta_{2, M}\right)\right]
\end{aligned}
$$

Finally, under 2-D stochastic assumptions, the unknown parameter vector is $\boldsymbol{\theta} \triangleq\left[\theta_{1,1}, \ldots, \theta_{1, M}, \theta_{2,1}, \ldots \theta_{2, M}, \operatorname{Re}\left\{R_{1,1}\right\}, \ldots, \operatorname{Re}\left\{R_{N, N}\right\}, \operatorname{Re}\left\{R_{1,2}\right\} \operatorname{Im}\left\{R_{1,2}\right\}, \ldots, \operatorname{Im}\left\{R_{N, N-1}\right\}\right]^{\mathrm{T}}$ consisting of $2 M+N^{2}$ unknowns, where $R_{i, j}$ is the $i, j$ th element of the signal covariance matrix. The $2 M \times 2 M$ upper-left corner of the inverse of the Fisher matrix $[8,12]$ corresponding to the parameters of direct interest is,

$$
\begin{aligned}
\mathbf{J}_{\mathrm{s}, 2}^{-1}= & \frac{\sigma_{n}^{2}}{2 K}\left(\operatorname{Re}\left\{\mathbf{D}^{\mathrm{H}}\left(\mathbf{I}-\mathbf{A}\left(\mathbf{A}^{\mathrm{H}} \mathbf{A}\right)^{-1} \mathbf{A}^{\mathrm{H}}\right) \mathbf{D} \odot\left(\mathbf{1}_{2} \mathbf{1}_{2}^{\mathrm{T}} \otimes \mathbf{U}\right)\right\}\right)^{-1} \\
& \mathbf{U}=\mathbf{P}\left(\mathbf{A}^{\mathrm{H}} \mathbf{A} \mathbf{P}+\sigma_{n}^{2} \mathbf{I}\right)^{-1} \mathbf{A}^{\mathrm{H}} \mathbf{A} \mathbf{P}
\end{aligned}
$$

with Das in (3.8). It can be shown that $\mathbf{U}=\mathbf{P A}{ }^{\mathrm{H}} \mathbf{R}^{-}$ ${ }^{1} \mathbf{A P}^{\mathrm{T}}$ [4] where $\boldsymbol{R}$ is defined in (3.6) so these UMA bounds are consistent.

\section{c. 1-D CRLB for the uniform linear array}

For the special case of a ULA with a single source, the bounds (3.4) and (3.6) simplify as follows. Assume all the elements are on the $x$-axis with spacing $\Delta_{x}$ meters, then,

$$
\begin{aligned}
\mathbf{a} & =\exp \left(-j \frac{2 \pi}{\lambda} \Delta_{x}[0,1, \ldots, N-1]^{\mathrm{T}} \cos (\alpha)\right) \\
\mathbf{d} & =-j \frac{2 \pi}{\lambda} \Delta_{x}[0,1, \ldots, N-1]^{T} \sin (\alpha) \odot \mathbf{a} \\
\mathbf{P}^{\mathrm{T}} & =P_{1} \\
\mathrm{~J}_{\mathrm{d}, 1} & =\frac{2 K P_{1}}{\sigma_{n}^{2}} \operatorname{Re}\left\{\left(-j \frac{2 \pi}{\lambda} \Delta_{x} \sin (\alpha) \mathbf{a}^{\mathrm{H}} \boldsymbol{\Lambda}_{[0: N-1]}\right)\left(\mathrm{I}-\frac{1}{M} \mathbf{a}^{\mathrm{H}}\right)\left(-j \frac{2 \pi}{\lambda} \Delta_{x} \sin (\alpha) \boldsymbol{\Lambda}_{[0: N-1]} \mathbf{a}\right)\right\} \\
& =\frac{2 K P_{1}}{\sigma_{n}^{2}}\left(\frac{2 \pi}{\lambda} \Delta_{x} \sin (\alpha)\right)^{2}\left(\mathbf{a}^{\mathrm{H}} \boldsymbol{\Lambda}_{[0: N-1][} \boldsymbol{\Lambda}_{[0: N-1]} \mathbf{a}-\frac{1}{M}\left(\mathbf{a}^{\mathrm{H}} \boldsymbol{\Lambda}_{[0: N-1]} \mathbf{a}^{2}\right)\right.
\end{aligned}
$$

where $\Lambda_{\mathrm{x}} \triangleq \operatorname{diag}(\mathbf{x})$. Then noting,

$$
\begin{aligned}
& \mathbf{a}^{\mathrm{H}} \boldsymbol{\Lambda}_{[0: N-1]} \mathbf{a}=\sum_{n=0}^{N-1} e^{-j c n} e^{+j c n} n=\sum_{n=0}^{N-1} n=\frac{N(N-1)}{2} \\
& \mathbf{a}^{\mathrm{H}} \boldsymbol{\Lambda}_{[0: N-1]} \boldsymbol{\Lambda}_{[0: N-1]} \mathbf{a}=\sum_{n=0}^{N-1} e^{-j c n} e^{+j c n} n^{2}=\sum_{n=0}^{N-1} n^{2}=\frac{N(N-1)(2 N-1)}{6}
\end{aligned}
$$




$$
\begin{aligned}
& J_{\mathrm{d}, 1}=\frac{2 K P_{1}}{\sigma_{n}^{2}}\left(\frac{2 \pi}{\lambda} \Delta_{x} \sin (\alpha)\right)^{2} \frac{1}{6}\left(0.5 N^{3}+2 N^{2}-2.5 N\right) \\
& J_{\mathrm{d}, 1}^{-1} \simeq \frac{6 K}{\operatorname{SNR}\left(2 \pi \Delta_{x} \sin (\alpha) / \lambda\right)^{2} \mathrm{~N}^{3}}
\end{aligned}
$$

where $c \triangleq 2 \pi \frac{\Delta_{x}}{\lambda} \sin (\alpha)$ and SNR $=P_{1} / \sigma_{n}{ }^{2}$. Only the third-order term for $N$ is included in the approximation (3.13) which agrees exactly with [3]. If we repeat this with the UMA (3.6), the difference lies in the term,

$$
P A^{\mathrm{H}} R^{-1} A P^{\mathrm{T}}=P A^{\mathrm{H}}(A P A+\sigma I)^{-1} A P^{\mathrm{T}}
$$

which becomes,

$$
\begin{aligned}
& P_{1}^{2} \mathbf{a}^{\mathrm{H}}\left(P_{1} \mathbf{a}^{\mathrm{H}}+\sigma_{n}^{2} I\right)^{-1} \mathbf{a}=P_{1}^{2} \mathbf{a}^{\mathrm{H}} \mathbf{a}\left[P_{1} N+\sigma_{n}^{2}\right]^{-1} \\
& \quad=\frac{P_{1}^{2} N}{P_{1} N+\sigma_{n}^{2}}
\end{aligned}
$$

where the r.h.s. of the first line acknowledges that for the single-user case, steering vector $\mathbf{a}$ is an eigenvector of $\mathbf{R}$. The final expression is,

$$
J_{s, 1}^{-1}=\frac{6 K \sigma_{n}^{2}}{\left(2 \pi \frac{\Delta_{x}}{\lambda} \sin (\alpha)\right)^{2} N^{3}\left(\frac{P_{1}^{2} N}{P_{1} N+\sigma_{n}^{2}}\right)}
$$

For $P_{1} N \gg \sigma_{n}^{2}$, the right-hand term in the denominator converges to $P_{1}$; hence, the CMA and UMA expressions are asymptotically equal (equal in the limit as SNR goes to infinity).

\section{d. Array definitions}

The bounds and procedures developed in this article can be used with arrays of arbitrary shape; sensor positions for a given array are specified by the vectors $\mathbf{p}_{x}, \mathbf{p}_{y}, \mathbf{p}_{z}$. However, our numerical examples focus mainly on the LSA, and a 3-D extension (" $L+z^{\prime \prime}$ array) defined in Figure 2. A Uniform Cross Array (UXA) is sometimes assumed instead of the LSA, however, the LSA gives better accuracy than the UXA for the same spacing and number of elements [17]. Practical size constraints may make the $L+z$ array preferable to the 2LSA.

An array may be decomposed into sub-arrays for "separate" processing. The sensor positions for the qth sub-array are specified by $\mathbf{p}_{q, x}, \mathbf{p}_{q}, y, \mathbf{p}_{q}, z$. We will assume that the L-shaped array is decomposed into subarrays consisting of the $x$-axis and $y$-axis elements (the central element is contained in both sub-arrays); the $L+$ $z$ array is decomposed into sub-arrays containing the " $x$ and $z^{\prime \prime}$ and " $y$ and $z$ " elements, respectively (all elements on $z$-axis are contained in both sub-arrays). The case of $L+z$ separated into three ULAs will not be considered, as the redundant measurements would need to be combined in some ad hoc way.

\section{e. Change of variables}

To map the 2-D angular CRLBs to bounds on the $(x, y)$ positions, we use the change of variables (2.5) or (2.6) in (3.2). For the "el-el" case we find the required derivatives as,

$$
\begin{aligned}
\frac{\mathrm{d} x_{i}}{\mathrm{~d} \alpha_{i}}= & -z\left(\sin \left(\alpha_{i}\right)\left(1-\cos ^{2}\left(\alpha_{i}\right)-\cos ^{2}\left(\beta_{i}\right)\right)^{-1 / 2}\right. \\
& \left.+\cos ^{2}\left(\alpha_{i}\right) \sin \left(\alpha_{i}\right)\left(1-\cos ^{2}\left(\alpha_{i}\right)-\cos ^{2}\left(\beta_{i}\right)\right)^{-3 / 2}\right) \\
\frac{\mathrm{d} x_{i}}{\mathrm{~d} \beta_{i}}= & -z\left(\cos \left(\alpha_{i}\right) \cos \left(\beta_{i}\right) \sin \left(\beta_{i}\right)\left(1-\cos ^{2}\left(\alpha_{i}\right)-\cos ^{2}\left(\beta_{i}\right)\right)^{-3 / 2}\right) \\
\frac{\mathrm{d} y_{i}}{\mathrm{~d} \alpha_{i}}= & -z\left(\cos \left(\beta_{i}\right) \cos \left(\alpha_{i}\right) \sin \left(\alpha_{i}\right)\left(1-\cos ^{2}\left(\alpha_{i}\right)-\cos ^{2}\left(\beta_{i}\right)\right)^{-3 / 2}\right) \\
\frac{\mathrm{d} y_{i}}{\mathrm{~d} \beta_{i}}= & -z\left(\sin \left(\beta_{i}\right)\left(1-\cos ^{2}\left(\alpha_{i}\right)-\cos ^{2}\left(\beta_{i}\right)\right)^{-1 / 2}\right. \\
& \left.+\cos ^{2}\left(\beta_{i}\right) \sin \left(\beta_{i}\right)\left(1-\cos ^{2}\left(\alpha_{i}\right)-\cos ^{2}\left(\beta_{i}\right)\right)^{-3 / 2}\right)
\end{aligned}
$$

and for the "az-el" case as,

$$
\begin{aligned}
& \frac{\mathrm{d} x_{i}}{\mathrm{~d} \theta_{i}}=-z \sin \left(\theta_{i}\right) \tan \left(\phi_{i}\right) \\
& \frac{\mathrm{d} x_{i}}{\mathrm{~d} \phi_{i}}=-z \cos \left(\theta_{i}\right) / \cos ^{2}\left(\phi_{i}\right) \\
& \frac{\mathrm{d} y_{i}}{\mathrm{~d} \theta_{i}}=-z \cos \left(\theta_{i}\right) \tan \left(\phi_{i}\right) \\
& \frac{\mathrm{d} y_{i}}{\mathrm{~d} \phi_{i}}=-z \sin \left(\theta_{i}\right) / \cos ^{2}\left(\phi_{i}\right)
\end{aligned}
$$

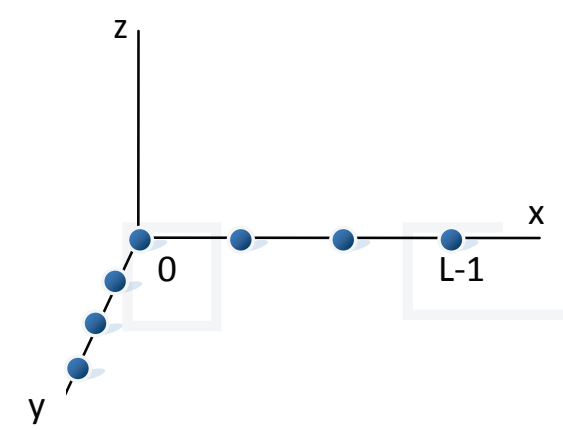

L-shaped Array (2L-1 elements)

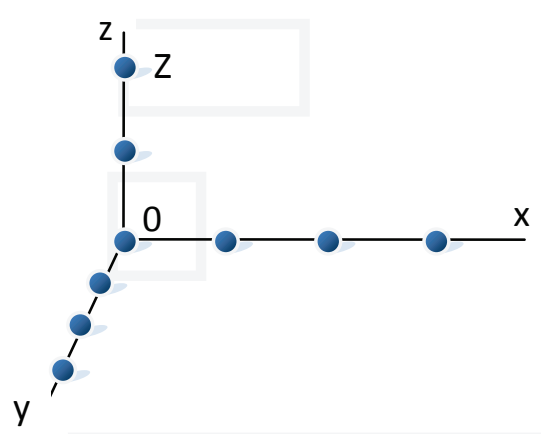

L+Z Shaped Array (2L+Z-1 elements) 
The values from (3.16) or (3.17) are inserted into the matrix $\dot{\mathrm{F}}$. Note that for our transformations, all terms $\mathrm{d} w_{i} / \mathrm{d} \theta_{j}$ are equal to zero for $i \neq j$, and the matrix $\dot{\mathbf{F}}$ will generally have only two non-zero entries per row or column.

Note that the new CRLB matrix of (3.2) will contain the $(x, y)$ error bounds for all the sources. From this we extract the $(2 \times 2)$ matrix for the user of interest, and this becomes the covariance used for drawing concentration ellipses described in Section 2b. The joint bound on $(x, y)$ will not depend on the choice of angular coordinate system.

\section{Separate versus joint bounds}

\section{a. Joint bounds}

The joint CRLB is computed in a straightforward way, using all the available sensors. Assume that an ordering of the elements is defined, and that $\mathbf{p}_{x}, \mathbf{p}_{y}, \mathbf{p}_{z}$ give the $x$, $y$, and $z$ coordinates (in meters) of elements 1 through $N$, relative to the array axes.

For the case of el-el coordinates, the $m$ th source has array response vector,

$$
\mathbf{a}\left(\alpha_{m}, \beta_{m}\right)=\exp \left(-j \frac{2 \pi}{\lambda}\left(\mathbf{p}_{x} \cos \left(\alpha_{m}\right)+\mathbf{p}_{y} \cos \left(\beta_{m}\right)+\mathbf{p}_{z}\left(1-\cos ^{2}\left(\alpha_{m}\right)-\cos ^{2}\left(\beta_{m}\right)\right)^{1 / 2}\right)\right)(4
$$

The array response matrix is,

$$
\mathbf{A}(\boldsymbol{\alpha}, \boldsymbol{\beta})=\left[\mathbf{a}\left(\alpha_{1}, \beta_{1}\right), \cdots, \mathbf{a}\left(\alpha_{M}, \beta_{M}\right)\right]
$$

The derivatives with respect to the estimated parameters are,

$$
\begin{aligned}
\mathbf{d}_{m, \alpha} & =\frac{\mathrm{d}}{\mathrm{d} \alpha_{m}} \mathbf{a}\left(\alpha_{m}, \beta_{m}\right) \\
& =j \frac{j \pi}{\lambda}\left(\mathbf{p}_{x} \sin \left(\alpha_{m}\right)-0.5 \mathbf{p}_{z}\left(1-\cos ^{2}\left(\alpha_{m}\right)-\cos ^{2}\left(\beta_{m}\right)\right)^{-1 / 2} \sin (2 \alpha)\right) \odot \mathbf{a}\left(\alpha_{m}, \beta_{m}\right) \\
\mathbf{d}_{m, \beta} & =\frac{\mathrm{d}}{\mathrm{d} \beta_{m}} \mathbf{a}\left(\alpha_{m}, \beta_{m}\right) \\
& =j \frac{2 \pi}{\lambda}\left(\mathbf{p}_{y} \sin \left(\beta_{m}\right)-0.5 \mathbf{p}_{z}\left(1-\cos ^{2}\left(\alpha_{m}\right)-\cos ^{2}\left(\beta_{m}\right)\right)^{-1 / 2} \sin (2 \beta)\right) \odot \mathbf{a}\left(\alpha_{m}, \beta_{m}\right)
\end{aligned}
$$

The derivative matrix is,

$$
\mathbf{D}(\boldsymbol{\alpha}, \boldsymbol{\beta})=\left[\mathbf{d}_{1, \alpha}, \cdots, \mathbf{d}_{M, \alpha}, \mathbf{d}_{1, \beta}, \cdots, \mathbf{d}_{M, \beta}\right]
$$

Using (4.2), (4.4), we can compute the deterministic bound (3.8) and the stochastic bound (3.9).

For the case of az-el coordinates, $m$ th source has array response vector,

$$
\mathbf{a}\left(\theta_{m}, \varphi_{m}\right)=\exp \left(-j \frac{2 \pi}{\lambda}\left(\mathbf{p}_{x} \sin \left(\varphi_{m}\right) \cos \left(\theta_{m}\right)+\mathbf{p}_{y} \sin \left(\varphi_{m}\right) \sin \left(\theta_{m}\right)+\mathbf{p}_{z} \cos \left(\varphi_{m}\right)\right)\right)
$$

and the derivatives are,

$$
\begin{aligned}
\mathbf{d}_{m, \theta} & =\frac{\mathrm{d}}{\mathrm{d} \theta_{m}} \mathbf{a}\left(\theta_{m}, \phi_{m}\right) \\
& =j \frac{2 \pi}{\lambda}\left(\mathbf{p}_{x} \sin \left(\phi_{m}\right) \sin \left(\theta_{m}\right)-\mathbf{p}_{y} \sin \left(\phi_{m}\right) \cos \left(\theta_{m}\right)\right) \mathbf{a}\left(\theta_{m}, \phi_{m}\right) \\
\mathbf{d}_{m, \phi} & =\frac{\mathrm{d}}{\mathrm{d} \phi_{m}} \mathbf{a}\left(\theta_{m}, \phi_{m}\right) \\
& =j \frac{2 \pi}{\lambda}\left(-\mathbf{p}_{x} \cos \left(\phi_{m}\right) \cos \left(\theta_{m}\right)-\mathbf{p}_{y} \cos \left(\phi_{m}\right) \sin \left(\theta_{m}\right)-\mathbf{p}_{z} \sin \left(\phi_{m}\right)\right) \odot \mathbf{a}\left(\theta_{m}, \phi_{m}\right)
\end{aligned}
$$

From these we find $\mathbf{A}(\theta, \phi), \mathbf{D}(\theta, \phi)$ as before, and compute the deterministic and stochastic bounds.

\section{b. Separate bounds}

Assume that the 2-D angle estimation is divided into two separate steps, with each 1-D angle computed from a subset of array elements, or sub-array. The separate bound cannot be lower than the joint bound, as any information about angle 2 that appears in sub-array 1 is ignored, and vice versa. Likewise, any noise correlation due to common elements in the sub-arrays will be ignored. Separate processing is of interest as it may lower the computational complexity of the angle estimation algorithm (performing two, 1-D searches may be much easier than performing a single 2-D search). Hardware savings are also possible if the radio frequency (RF) chains (i.e., amplifiers, mixers, filters, etc.) associated with the sensors are shared among the sub-arrays, reducing the overall cost, size, weight, and power of the system. For example, $M$ sensors will only require $M / 2 \mathrm{RF}$ chains if a switching mechanism is used and only one sub-array collects data at a time. As the system spends half the normal observation time collecting samples from each sub-array, and the below bounds would be modified by simply dividing the number of snapshots by 2 (our results do not include this factor of 2). The separate bound will enable us determine the performance cost of "separate" processing relative to the full (joint) array processing.

To form the separate bound, first we define the elements within each sub-array. Then we find the CRLB under conditions where one angle is being estimated, while the other is unknown. Then, the two resulting Fisher matrices are combined into a single, larger $2 M$-by- $2 M$ matrix. Let $\mathrm{p}_{q, x}, \mathrm{p}_{q, y}, \mathrm{p}_{q, z}$ denote the $x, y, z$ coordinates of elements in the $q$ th sub-array, $q=1,2$. Assume that the first sub-array will estimate $\boldsymbol{\theta}_{1}$ with $\boldsymbol{\theta}_{2}$ as an unknown, deterministic nuisance variable, and likewise the second sub-array estimates $\boldsymbol{\theta}_{2}$ with $\boldsymbol{\theta}_{1}$ as an unknown. According to CRLB theory, we must treat both $\boldsymbol{\theta}_{1}$ and $\boldsymbol{\theta}_{2}$ as unknowns to be estimated, but use only the CRLB sub-matrix corresponding to the variable being estimated $[2,15]$. Let $\mathbf{J}_{\mathrm{t}, 2}(q)$ denote the Fisher matrix (3.8), (3.9) using only the elements of the $q$ th sub-array. Define the "separate" CRLB as

$$
\mathbf{J}_{t, \text { Sep }}^{-1}=\left[\begin{array}{cc}
\mathbf{W} & \mathbf{0}_{M} \\
\mathbf{0}_{M} & \mathbf{V}
\end{array}\right], \quad \mathbf{W}=\left.\mathbf{J}_{\mathrm{t}, 2}^{-1}(1)\right|_{\mathrm{UL}}, \quad \mathbf{V}=\left.\mathbf{J}_{\mathrm{t}, 2}^{-1}(2)\right|_{\mathrm{LR}}
$$

where $t$ (type) may be either s (stochastic bound) or d (deterministic bound), and "UL" and "LR" denote extracting the upper-left and lower-right quadrants, 
respectively. Note that the block diagonal form of (4.7) results directly from making independent angle estimates from the sub-arrays-any beneficial correlation between the angles has been lost. Starting with the joint bound in the deterministic case,

$$
\mathbf{J}_{\mathrm{d}, 2}=\frac{2 K}{\sigma_{n}^{2}}\left[\begin{array}{ll}
\operatorname{Re}\left\{\mathbf{D}_{1}^{\mathrm{H}} \mathbf{A}^{\perp} \mathbf{D}_{1} \odot \mathbf{P}^{\mathrm{T}}\right\} & \operatorname{Re}\left\{\mathbf{D}_{1}^{\mathrm{H}} \mathbf{A}^{\perp} \mathbf{D}_{2} \odot \mathbf{P}^{\mathrm{T}}\right\} \\
\operatorname{Re}\left\{\mathbf{D}_{2}^{\mathrm{H}} \mathbf{A}^{\perp} \mathbf{D}_{1} \odot \mathbf{P}^{\mathrm{T}}\right\} & \operatorname{Re}\left\{\mathbf{D}_{2}^{\mathrm{H}} \mathbf{A}^{\perp} \mathbf{D}_{2} \odot \mathbf{P}^{\mathrm{T}}\right\}
\end{array}\right](
$$

where $\mathbf{A}^{\perp} \triangleq \mathbf{I}-\mathbf{A}\left(\mathbf{A}^{\mathrm{H}} \mathbf{A}\right)^{-1} \mathbf{A}^{\mathrm{H}}$ then using the standard block inversion formulas [18], we can find the UL and LR quantities desired in (4.7) as,

$$
\begin{aligned}
\mathbf{W}= & \frac{\sigma_{n}^{2}}{2 K}\left(\left(\operatorname{Re}\left\{\mathbf{D}_{1} \mathbf{A}^{\perp} \mathbf{D}_{1} \odot\right\} \mathbf{P}^{\mathrm{T}}\right)-\left(\operatorname{Re}\left\{\mathbf{D}_{1} \mathbf{A}^{\perp} \mathbf{D}_{2} \odot \mathbf{P}^{\mathrm{T}}\right\}\right)\right. \\
& \left.\left(\operatorname{Re}\left\{\mathbf{D}_{2} \mathbf{A}^{\perp} \mathbf{D}_{2} \odot \mathbf{P}^{\mathrm{T}}\right\}\right)^{-1}\left(\operatorname{Re}\left\{\mathbf{D}_{2} \mathbf{A}^{\perp} \mathbf{D}_{1} \odot \mathbf{P}^{\mathrm{T}}\right\}\right)\right)^{-1} \\
\mathbf{V}= & \frac{\sigma_{n}^{2}}{2 K}\left(\left(\operatorname{Re}\left\{\mathbf{D}_{2} \mathbf{A}^{\perp} \mathbf{D}_{2} \odot \mathbf{P}^{\mathrm{T}}\right\}\right)-\left(\operatorname{Re}\left\{\mathbf{D}_{2} \mathbf{A}^{\perp} \mathbf{D}_{1} \odot \mathbf{P}^{\mathrm{T}}\right\}\right)\right. \\
& \left.\left(\operatorname{Re}\left\{\mathbf{D}_{1} \mathbf{A}^{\perp} \mathbf{D}_{1} \odot \mathbf{P}^{\mathrm{T}}\right\}\right)^{-1}\left(\operatorname{Re}\left\{\mathbf{D}_{1} \mathbf{A}^{\perp} \mathbf{D}_{2} \odot \mathbf{P}^{\mathrm{T}}\right\}\right)\right)^{-1}
\end{aligned}
$$

Breaking the array into non-linear sub-arrays may make it difficult to estimate one angle while the other is unknown, hence complexity may not be reduced. However, if the sub-arrays are linear arrays, then each is only sensitive to $\alpha$ (or, $\beta$ ) and ordinary 1-D estimation can be used to reduce the overall complexity. In the case of a linear sub-array, the 2-D CRLB does not exist (i.e., the Fisher matrix is not invertible, as you cannot estimate two angles from a single linear array); so, we use the ordinary 1-D CRLB formulation from each sub-array,

$$
\mathbf{W}=\mathbf{J}_{\mathrm{t}, 1}^{-1}(1), \quad \mathbf{V}=\mathbf{J}_{\mathrm{t}, 1}^{-1}(2)
$$

\section{c. Separability conditions}

Generally, arrays are not "separable", meaning joint estimation will outperform separate estimation. However, in some special array geometries the bounds are "separable," and separate processing is optimal.

Theorem 1 The CRLB for a Uniform Cross Array (UCA) with a single user is separable.

This result is stated in [17] for the deterministic case without proof. For the UCA with $2 L-1$ elements ( $L$ assumed odd), the array position vectors are,

$$
\begin{gathered}
\mathbf{p}_{x} \triangleq\left[\mathbf{v}_{x}, \mathbf{0}_{1 \times(L-1)}\right]^{\mathrm{T}}, \mathbf{v}_{x}=\left[-N_{\mathrm{UCA}}, \ldots,-1,0,1, \ldots, N_{\mathrm{UCA}}\right] \\
N_{\mathrm{UCA}}=\frac{L-1}{2} \\
\mathbf{p}_{y} \triangleq\left[\mathbf{0}_{1 \times L}, \mathbf{v}_{y}\right]^{\mathrm{T}}, \mathbf{v}_{y}=\left[-N_{\mathrm{UCA}}, \ldots,-1,1, \ldots, N_{\mathrm{UCA}}\right]
\end{gathered}
$$

For the single user case,

$$
\begin{aligned}
& \mathbf{a}(\alpha, \beta)=\exp \left(-j \frac{2 \pi}{\lambda} \Delta_{x}\left(\mathbf{p}_{x} \cos (\alpha)+\mathbf{p}_{y} \cos (\beta)\right)\right) \in C^{2 L-1 \times 1} \\
& \mathbf{A}^{\perp}=\mathbf{I}-\mathbf{a}\left(\mathbf{a}^{\mathrm{H}} \mathbf{a}\right)^{-1} \mathbf{a}^{\mathrm{H}}=\mathbf{I}-\frac{1}{2 L-1} \mathbf{a ~ a ~}^{\mathrm{H}} \\
& \mathbf{d}_{\alpha}=j \frac{2 \pi}{\lambda} \Delta_{x} \mathbf{p}_{x} \sin (\alpha) \odot \mathbf{a}(\alpha, \beta)=j \frac{2 \pi}{\lambda} \Delta_{x} \sin (\alpha) \Lambda_{p x} \mathbf{a}(\alpha, \beta) \\
& \mathbf{d}_{\beta}=j \frac{2 \pi}{\lambda} \Delta_{x} \mathbf{p}_{\gamma} \sin (\beta) \odot \mathbf{a}(\alpha, \beta)=j \frac{2 \pi}{\lambda} \Delta_{x} \sin (\beta) \Lambda_{p \gamma} \mathbf{a}(\alpha, \beta)
\end{aligned}
$$

where $\Lambda_{\mathrm{x}} \triangleq \operatorname{diag}(\mathbf{x})$ is a diagonal matrix. Inserting into (4.8), we want to prove that the resulting matrix has the form (4.7). Beginning with the upper-right term,

$$
\begin{aligned}
& \mathbf{D}_{1}^{\mathrm{H}} \mathbf{A}^{\perp} \mathbf{D}_{2}=\mathbf{a}^{\mathrm{H}} \boldsymbol{\Lambda}_{\rho x}\left(\mathbf{I}-\frac{1}{2 L-1} \mathbf{a} \mathbf{a}^{\mathrm{H}}\right) \boldsymbol{\Lambda}_{\rho \gamma} \mathbf{a}=\mathbf{a}^{\mathrm{H}} \boldsymbol{\Lambda}_{\rho x} \boldsymbol{\Lambda}_{\rho \gamma \mathbf{a}} \mathbf{a}-\frac{1}{2 L-1}\left(\mathbf{a}^{\mathrm{H}} \boldsymbol{\Lambda}_{\rho x \mathrm{x}} \mathbf{a}\right)\left(\mathbf{a}^{\mathrm{H}} \boldsymbol{\Lambda}_{\rho \gamma} \mathbf{a}\right) \stackrel{?}{=} \mathbf{0} \\
& =0-\left(\mathbf{a}^{\mathrm{H}} \boldsymbol{\Lambda}_{p x} \mathbf{a}\right)\left(\mathbf{a}^{\mathrm{H}} \boldsymbol{\Lambda}_{p \gamma} \mathbf{a}\right) \\
& \left(\mathbf{a}^{\mathrm{H}} \boldsymbol{\Lambda}_{p \mathbf{x}} \mathbf{x}\right)=\sum_{i=1}^{2 L-1} e^{j f(i)} e^{-i f(i)} p_{x}(i)=\operatorname{sum}\left(\mathbf{p}_{x}\right)=\operatorname{sum}\left(\mathbf{v}_{x}\right)=0
\end{aligned}
$$

Where $f(i)$ is a real-valued function corresponding to the exponent of the array response vector, the fact that $\Lambda_{p x} \Lambda_{p y}=\mathbf{0}$, and that the sum over $\mathbf{v}_{x}$ is zero by inspection. Now turning to the upper-left term, and defining $\mathbf{a} \triangleq\left[\mathbf{a}_{1}^{\mathrm{T}}, \mathbf{a}_{2}^{\mathrm{T}}\right]^{\mathrm{T}}$,

$$
\mathbf{D}_{1}^{\mathrm{H}} \mathbf{A}^{\perp} \mathbf{D}_{1}=\left(j 2 \pi \frac{\Delta_{x}}{\lambda} \cos \alpha\right)^{2} \mathbf{a}_{1}^{\mathrm{H}} \boldsymbol{\Lambda}_{v x}\left(\mathbf{I}_{L}-\frac{1}{2 L-1} \mathbf{a}_{1} \mathbf{a}_{1}^{\mathrm{H}}\right) \boldsymbol{\Lambda}_{v x} \mathbf{a}_{1}
$$

By inspection, this term is equal to the 1-D bound $J_{1, \mathrm{~d}}$ (1) except for the scale factor $2 L-1$. However, for the special case under consideration, this term is zero because $\mathbf{a}_{1}^{\mathrm{H}} \boldsymbol{\Lambda}_{v x} \mathbf{a}_{1}=\operatorname{sum}\left(\mathbf{v}_{x}\right)=0$.

Likewise, we may show that the lower-left term is zero and the lower-right term is $J_{1, \mathrm{~d}}(2)$ which completes the proof in the deterministic case. However, as only properties of $\mathbf{D}^{\mathrm{H}} \mathbf{A}^{\perp} \mathbf{D}$ were used, the result extends immediately to the stochastic case as well.

Theorem 2 The CRLB for an L-shaped Array (LSA) with a single user is not separable.

For the LSA with $2 L-1$ elements, the element position vectors are

$$
\begin{aligned}
& \mathbf{p}_{x} \triangleq\left[\mathbf{u}_{x}, \mathbf{0}_{1 \times(L-1)}\right]^{\mathrm{T}}, \quad \mathbf{u}_{x}=[0,1, \cdots, L-1] \\
& \mathbf{p}_{\gamma} \triangleq\left[\mathbf{0}_{1 \times L}, \mathbf{u}_{y}\right]^{\mathrm{T}}, \quad \mathbf{u}_{y}=[1, \cdots, L-1]
\end{aligned}
$$

Examining the term $\mathbf{D}_{1}^{\mathrm{H}} \mathbf{A}^{\perp} \mathbf{D}_{2}$, as in the upper-right term of (4.8),

$$
\begin{aligned}
& \left(2 \pi \frac{d}{\lambda}\right)^{2} \cos \alpha \cos \beta \mathbf{a}^{\mathrm{H}} \boldsymbol{\Lambda}_{x}\left(\mathrm{I}-\frac{1}{2 L-1} \mathbf{a}^{\mathrm{H}}\right) \boldsymbol{\Lambda}_{\gamma} \mathbf{a} \\
& =\left(2 \pi \frac{d}{\lambda}\right)^{2} \cos \alpha \cos \beta\left(-\frac{1}{2 L-1}\right)\left(\mathbf{a}^{\mathrm{H}} \boldsymbol{\Lambda}_{x} \mathbf{a}\right)\left(\mathbf{a}^{\mathrm{H}} \boldsymbol{\Lambda}_{\gamma} \mathbf{a}\right) \\
& \left(\mathbf{a}^{\mathrm{H}} \boldsymbol{\Lambda}_{x} \mathbf{a}\right)=\left[\mathbf{a}_{1}^{\mathrm{H}}, \mathbf{a}_{2}^{\mathrm{H}}\right]\left[\begin{array}{cr}
\operatorname{diag}\left(\mathbf{u}_{x}\right) & 0 \\
\mathbf{0} & \mathbf{0}
\end{array}\right]\left[\begin{array}{l}
\mathbf{a}_{1} \\
\mathbf{a}_{2}
\end{array}\right]=\mathbf{a}_{1}^{\mathrm{H}} \operatorname{diag}\left(\mathbf{u}_{x}\right) \mathbf{a}_{1}=\operatorname{sum}\left(\mathbf{u}_{x}\right) \neq 0 \\
& \text { Likewise, }\left(\mathbf{a}^{\mathrm{H}} \boldsymbol{\Lambda}_{\gamma} \mathbf{a}\right) \neq 0
\end{aligned}
$$

Since the upper-right is not zero, (4.7) does not hold for either UMA or CMA. 
An intuitive explanation for Theorem 2 is to note that the LSA is able to make measurements over a "long baseline" pair-the pair of sensors at the tips of the two arms. There are no two elements within a sub-array having as large a spacing as this, giving an advantage to the joint processing for LSA. By contrast, the tip-to-tip distance in the UCA does not exceed the largest spacing found in a sub-array, and there is no such advantage.

Theorem 3 The 2-D CRLB is non-increasing in the number of sensors (regardless of their positions).

This is an extension to the 2-D case of a proof in [3] for the deterministic case. Consider a system with $N$ sensors, $\mathbf{A} \in C^{N \times M}, \mathbf{D} \in C^{N \times 2 M}$, and the Fisher matrix,

$$
\mathbf{J}=\frac{\sigma_{n}^{2}}{2 K}\left[\begin{array}{lll}
\operatorname{Re}\left\{\left(\mathbf{D}_{\alpha}^{\mathrm{H}} \mathbf{A}^{\perp} \mathbf{D}_{\alpha}\right) \odot \mathbf{P}^{\mathrm{T}}\right\} & \operatorname{Re}\left\{\left(\mathbf{D}_{\beta}^{\mathrm{H}} \mathbf{A}^{\perp} \mathbf{D}_{\alpha}\right) \odot \mathbf{P}^{\mathrm{T}}\right\} \\
\operatorname{Re}\left\{\left(\mathbf{D}_{\alpha}^{\mathrm{H}} \mathbf{A}^{\perp} \mathbf{D}_{\beta}\right) \odot \mathbf{P}^{\mathrm{T}}\right\} & \operatorname{Re}\left\{\left(\mathbf{D}_{\beta}^{\mathrm{H}} \mathbf{A}^{\perp} \mathbf{D}_{\beta}\right) \odot \mathbf{P}^{\mathrm{T}}\right\}
\end{array}\right]
$$

Adding an extra sensor,

$$
\overline{\mathbf{A}}=\left[\begin{array}{c}
\mathbf{A} \\
\mathbf{u}^{\mathrm{T}}
\end{array}\right], \quad \overline{\mathbf{D}}=\left[\begin{array}{ll}
\overline{\mathbf{D}}_{\alpha} & \overline{\mathbf{D}}_{\beta}
\end{array}\right], \quad \overline{\mathbf{D}}_{\alpha}=\left[\begin{array}{c}
\mathbf{D}_{\alpha} \\
\mathbf{v}_{\alpha}^{\mathrm{T}}
\end{array}\right]
$$

The new system (with over-bar notation) has Fisher matrix,

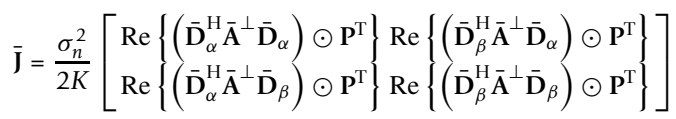

Note that,

$$
\begin{aligned}
& \overline{\mathbf{A}}^{\perp} \triangleq \mathbf{I}_{N+1}-\overline{\mathbf{A}}\left(\overline{\mathbf{A}}^{\mathrm{H}} \overline{\mathbf{A}}\right)^{-1} \overline{\mathbf{A}}^{\mathrm{H}} \\
& \quad=\left[\begin{array}{ll}
\mathbf{I}_{N} & 0 \\
\mathbf{0}^{\mathrm{T}} & 1
\end{array}\right]-\left[\begin{array}{c}
\mathbf{A} \\
\mathbf{u}^{\mathrm{T}}
\end{array}\right]\left(\mathbf{A}^{\mathrm{H}} \mathbf{A}+\mathbf{u}^{*} \mathbf{u}^{\mathrm{T}}\right)^{-1}\left[\mathbf{A}^{\mathrm{H}} \mathbf{u}^{*}\right] \\
& \left(\mathbf{A}^{\mathrm{H}} \mathbf{A}+\mathbf{u}^{*} \mathbf{u}^{\mathrm{T}}\right)^{-1}=\left(\mathbf{A}^{\mathrm{H}} \mathbf{A}\right)^{-1}-\frac{\left(\mathbf{A}^{\mathrm{H}} \mathbf{A}\right)^{-1} \mathbf{u}^{\mathrm{T}} \mathbf{u}^{*}\left(\mathbf{A}^{\mathrm{H}} \mathbf{A}\right)^{-1}}{1+\mathbf{u}^{\mathrm{T}}\left(\mathbf{A}^{\mathrm{H}} \mathbf{A}\right)^{-1} \mathbf{u}^{*}}=\left(\mathbf{A}^{\mathrm{H}} \mathbf{A}\right)^{-1}-\Sigma
\end{aligned}
$$

Focusing on the upper-left term of (4.17),

$$
\overline{\mathbf{D}}_{\alpha}^{\mathrm{H}} \overline{\mathbf{A}}^{\perp} \overline{\mathbf{D}}_{\alpha}=\left[\begin{array}{ll}
\mathbf{D}_{\alpha}^{\mathrm{H}} & \mathbf{v}_{\alpha}^{*}
\end{array}\right]\left(\left[\begin{array}{ll}
\mathbf{I}_{N} & 0 \\
0^{\mathrm{T}} & 1
\end{array}\right]-\left[\begin{array}{c}
\mathbf{A} \\
\mathbf{u}^{\mathrm{T}}
\end{array}\right]\left(\left(\mathrm{A}^{\mathrm{H}} \mathbf{A}\right)^{-1}-\Sigma\right)\left[\mathrm{A}^{\mathrm{H}} \mathbf{u}^{*}\right]\right)\left[\begin{array}{c}
\mathbf{D}_{\alpha} \\
\mathbf{v}_{\alpha}^{\mathrm{T}}
\end{array}\right]
$$

Following the somewhat involved algebra of [3, App. F] this can be simplified to,

$$
\overline{\mathbf{D}}_{\alpha}^{\mathrm{H}} \overline{\mathbf{A}}^{\perp} \overline{\mathbf{D}}_{\alpha}=\mathbf{D}_{\alpha}^{\mathrm{H}} \mathbf{A}^{\perp} \mathbf{D}_{\alpha}+\varepsilon_{\alpha} \varepsilon_{\alpha}^{\mathrm{H}}, \quad \varepsilon_{\alpha}=\frac{\mathbf{v}_{\alpha}^{*}-\mathbf{D}_{\alpha}^{\mathrm{H}} \mathbf{A}\left(\mathbf{A}^{\mathrm{H}} \mathbf{A}\right)^{-1} \mathbf{u}^{*}}{\sqrt{1+\mathbf{u}^{\mathrm{T}}\left(\mathbf{A}^{\mathrm{H}} \mathbf{A}\right)^{-1} \mathbf{u}^{*}}}
$$

Likewise, for the three remaining terms in (4.17) we can show,

$$
\begin{aligned}
& \overline{\mathbf{D}}_{\beta}^{\mathrm{H}} \overline{\mathbf{A}}^{\perp} \overline{\mathbf{D}}_{\alpha}=\mathbf{D}_{\beta}^{\mathrm{H}} \mathbf{A}^{\perp} \mathbf{D}_{\alpha}+\boldsymbol{\varepsilon}_{\beta} \boldsymbol{\varepsilon}_{\alpha}^{\mathrm{H}} \\
& \overline{\mathbf{D}}_{\alpha}^{\mathrm{H}} \overline{\mathbf{A}}^{\perp} \overline{\mathbf{D}}_{\beta}=\mathbf{D}_{\alpha}^{\mathrm{H}} \mathbf{A}^{\perp} \mathbf{D}_{\beta}+\boldsymbol{\varepsilon}_{\alpha} \boldsymbol{\varepsilon}_{\beta}^{\mathrm{H}} \\
& \overline{\mathbf{D}}_{\beta}^{\mathrm{H}} \overline{\mathbf{A}}^{\perp} \overline{\mathbf{D}}_{\beta}=\mathbf{D}_{\beta}^{\mathrm{H}} \mathbf{A}^{\perp} \mathbf{D}_{\beta}+\boldsymbol{\varepsilon}_{\beta} \boldsymbol{\varepsilon}_{\beta}^{\mathrm{H}}
\end{aligned}
$$

The Fisher matrix is now,

$$
\begin{aligned}
\overline{\mathbf{J}} & =\operatorname{Re}\left\{\left(\left[\begin{array}{l}
\mathbf{D}_{\alpha}^{\mathrm{H}} \mathbf{A}^{\perp} \mathbf{D}_{\alpha} \mathbf{D}_{\alpha}^{\mathrm{H}} \mathbf{A}^{\perp} \mathbf{D}_{\beta} \\
\mathbf{D}_{\beta}^{\mathrm{H}} \mathbf{A}^{\perp} \mathbf{D}_{\alpha} \mathbf{D}_{\beta}^{\mathrm{H}} \mathbf{A}^{\perp} \mathbf{D}_{\beta}
\end{array}\right]+\left[\begin{array}{l}
\boldsymbol{\varepsilon}_{\alpha} \\
\boldsymbol{\varepsilon}_{\beta}
\end{array}\right]\left[\begin{array}{l}
\boldsymbol{\varepsilon}_{\alpha} \\
\boldsymbol{\varepsilon}_{\beta}
\end{array}\right]^{\mathrm{H}}\right) \odot\left[\begin{array}{l}
\mathbf{P}^{\mathrm{T}} \mathbf{P}^{\mathrm{T}} \\
\mathbf{P}^{\mathrm{T}} \mathbf{P}^{\mathrm{T}}
\end{array}\right]\right\} \\
& =\mathbf{J}+\operatorname{Re}\left\{\left[\begin{array}{l}
\boldsymbol{\varepsilon}_{\alpha} \\
\boldsymbol{\varepsilon}_{\beta}
\end{array}\right]\left[\begin{array}{l}
\boldsymbol{\varepsilon}_{\alpha} \\
\boldsymbol{\varepsilon}_{\beta}
\end{array}\right] \odot\left[\begin{array}{l}
\mathbf{P}^{\mathrm{T}} \mathbf{P}^{\mathrm{T}} \\
\mathbf{P}^{\mathrm{T}} \mathbf{P}^{\mathrm{T}}
\end{array}\right]\right\} \triangleq \mathbf{J}+\operatorname{Re}\{\mathbf{E} \odot \overline{\mathbf{P}}\}
\end{aligned}
$$

The matrix $\overline{\mathbf{P}}$ is easily found to be positive semi-definite (psd) as follows. Since $\mathbf{P}_{\mathrm{T}}$ is a covariance matrix we can write it in terms of eigenvectors and eigenvalues as $\mathbf{P}^{\mathrm{T}}=\mathbf{V D V} \mathbf{V}^{\mathrm{H}}$. The matrix $\overline{\mathbf{P}}$ has $2 \mathrm{M}$ eigenvectors, which by inspection are $\overline{\mathbf{v}}=\left[\mathbf{v}_{i}^{\mathrm{T}}, \mathbf{v}_{i}^{\mathrm{T}}\right]^{\mathrm{T}},\left[\mathbf{v}_{i}^{\mathrm{T}},-\mathbf{v}_{i}^{\mathrm{T}}\right]^{\mathrm{T}}$, and the eigenvalues are $\bar{\lambda}_{i}=2 \lambda_{i}$ and $\bar{\lambda}_{i}=0$ (with multiplicity M). Hence, for $\mathbf{P}^{\mathrm{T}} \geq 0$, it follows that $\overline{\mathbf{P}} \geq 0$. Next, it follows from the Schur Product Theorem that $\mathbf{E} \odot \overline{\mathbf{P}} \geq 0$ since the individual matrices are psd. Then, $\mathbf{X} \geq 0 \Rightarrow \operatorname{Re}\{X\} \geq 0$ for $\mathbf{X}$ Hermitian. So, we can state, $\overline{\mathbf{J}} \geq \mathbf{J}$, and $\overline{\mathbf{J}}^{-1} \leq \mathbf{J}^{-1}$, which completes the proof for the deterministic case. To extend the result to the stochastic case, it is only necessary to prove that $\mathbf{U} \geq 0$, which follows directly from the factorization $\mathbf{U}=\left(\mathbf{R}^{-1 / 2} \mathbf{A}^{\mathrm{H}} \mathbf{P}\right)^{\mathrm{H}}\left(\mathbf{R}^{-1 / 2} \mathbf{A}^{\mathrm{H}} \mathbf{P}\right)$.

\section{Numerical results}

Unless otherwise stated, our numerical results assume a LSA of 11 elements $(L=6)$, spaced at half-wavelength and the CMA is used. The instantaneous SNR = $10 \mathrm{~dB}$, 15 snapshots are used, and we set $\mathrm{CF}=0.95$. The array height is either 1500 or $3000 \mathrm{ft}$; there is no fading or multipath. Figure 3 compares error ellipses for separate processing at two heights and two locations. In Figure $3 \mathrm{a}$ the source is almost under the array, at $(0.25,0.25)$ $\mathrm{km}$. The confidence contours are nearly circular, and notice the position error is smaller at lower height. On the other hand, Figure $3 \mathrm{~b}$ shows a more distant source at $(0.75,0.75) \mathrm{km}$. Now the position error is smaller at the greater height. This demonstrates a basic trade-off for our location geometry: distant sources require greater array height. Intuitively, for a distant source, lower height increases the error two ways: the source is increasingly off-boresight which reduces the effective aperture size, i.e., the $\sin (\alpha)$ dependence seen in (3.13), and simultaneously, the error contour expands like a "lengthening shadow" when projected to the ground plane.

Figure 4 shows the source at $(0.25,0.25)$ and height $=$ $1500 \mathrm{ft}$. The contours correspond to separate, and joint processing (with el-el and az-el coordinates). In Figure 4a there is no interferer; we see a modest performance gain with joint processing and the contours for joint elel and az-el are identical, as expected. However, in Figure $4 \mathrm{~b}$ an interferer (an independent, in-band source) is added at location $(0.1,-1.1) \mathrm{km}$ at $0 \mathrm{~dB}$ relative to the desired source. Now the ellipse for separate processing is greatly enlarged, and is much worse than joint 


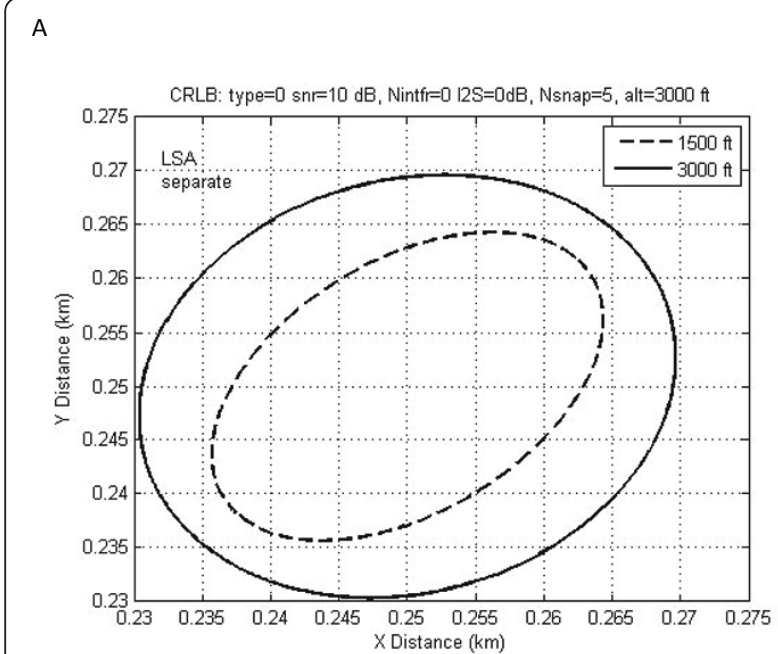

B

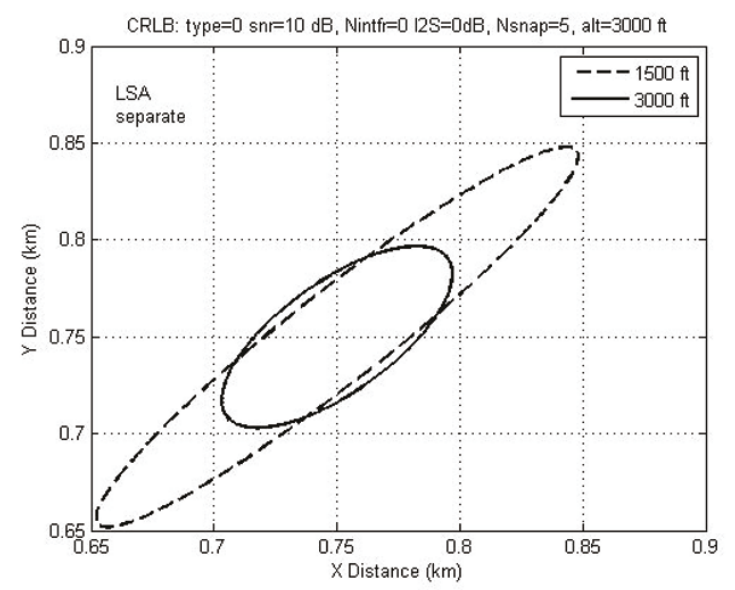

Figure 3 Separate processing at two heights (1500 and 3000 ft). (a) Source at $(0.25,0.25) \mathrm{km}$. (b) Source at $(0.75,0.75) \mathrm{km}$.

processing. As seen, joint processing seems particularly beneficial when multiple sources are present. We repeated Figure 4a,b under the UMA, and the results appeared equivalent (not shown).

In Figure 5, the interferer is moved to the location $(0.5,-1.95)$. Note that a linear array is subject to an "ambiguity cone": identical array responses are created by sources rotated around the array axis. Here, an original source at $(0.25,0.75)$ and another source anywhere along the line $c(0.25,-0.75)$ creates the same response. The interferer in Figure 5 is nearly on this line $(c=2)$ and the related $\mathbf{A}\left(\theta_{1}, \theta_{2}\right)$ matrix is ill-conditioned as both sources have nearly the same response vector. As seen, the separate approach produces a very large
A

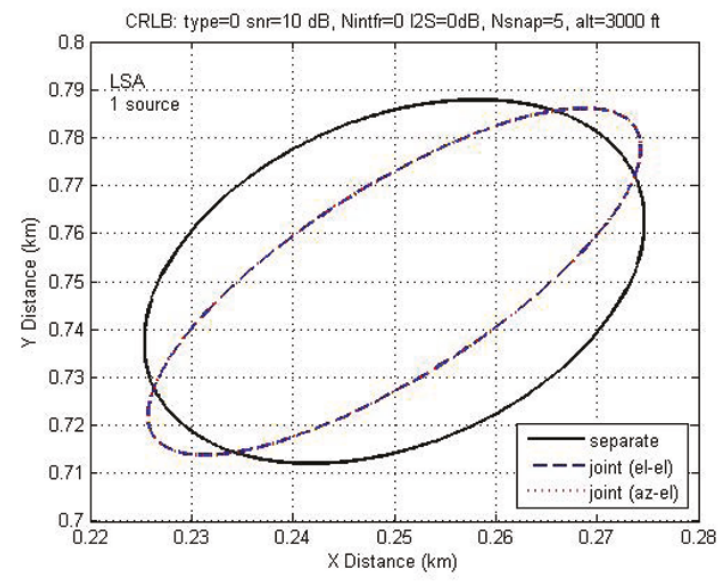

B

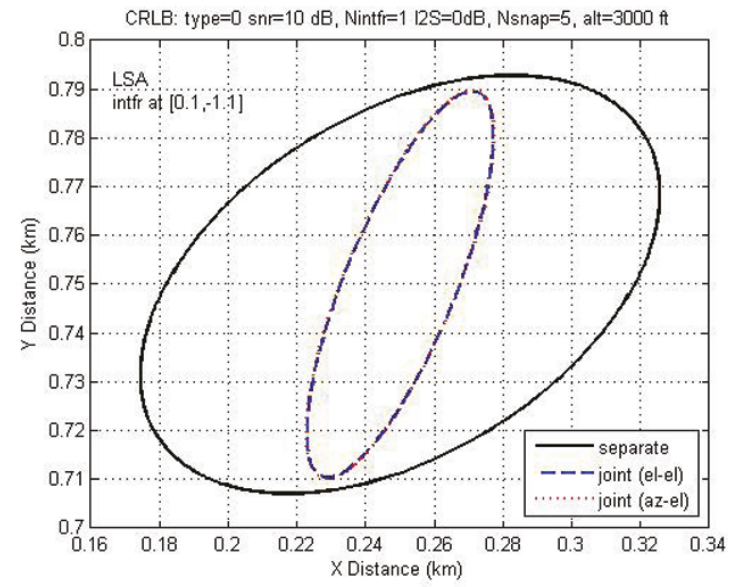

Figure 4 Separate and joint processing. (a) No interference: separate vs. joint, (b) One interferer: separate vs. joint.

ellipse. However, the joint approaches give excellent error performance, as in the larger space (with dimension $2 L$ - 1) the $\mathrm{A}\left(\theta_{1}, \theta_{2}\right)$ matrix is not ill-conditioned.

In Figure 6 we compare the LSA with the $L+z$ array having two additional elements on the $z$-axis $(z=2)$. In Figure $6 \mathrm{a}$, a single source is present. The LSA is shown with separate and joint processing as before. The $L+z$ with separate processing shows only a very small gain relative to LSA; however, joint processing shows a strong improvement. In Figure $6 \mathrm{~b}$, a single interferer is present, and some additional improvement between LSA and $L+z$ with separate processing is observed. Again, large gains are seen obtained for $L+z$ over LSA with joint processing. The "lengthening shadow" effect can be significantly reduced by joint processing of the $L+z$ array; it is reduced by about $50 \%$. 


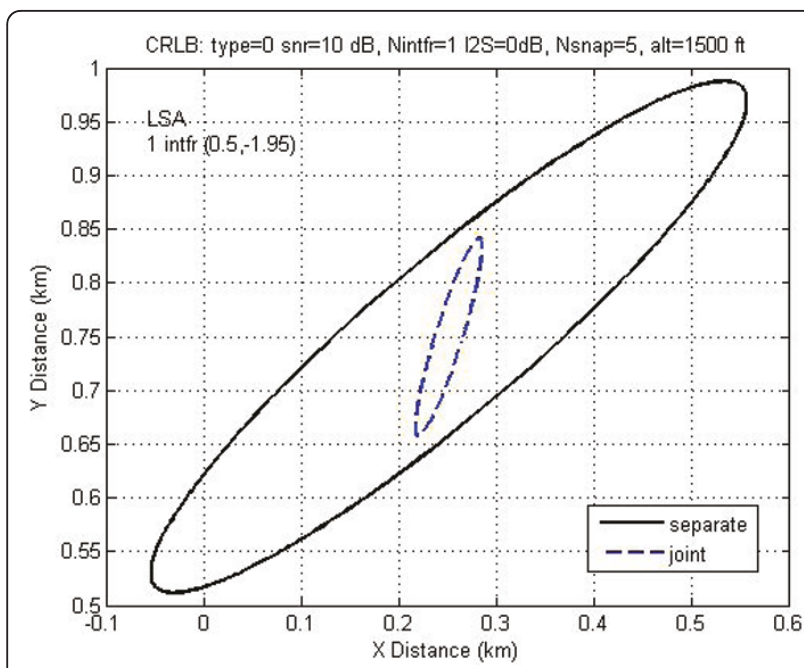

Figure 5 L-array with a single interferer.

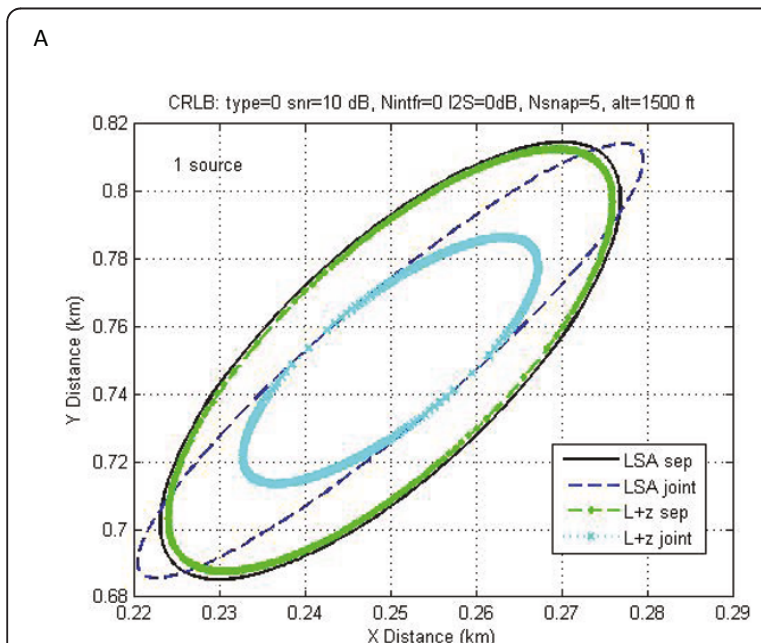

B

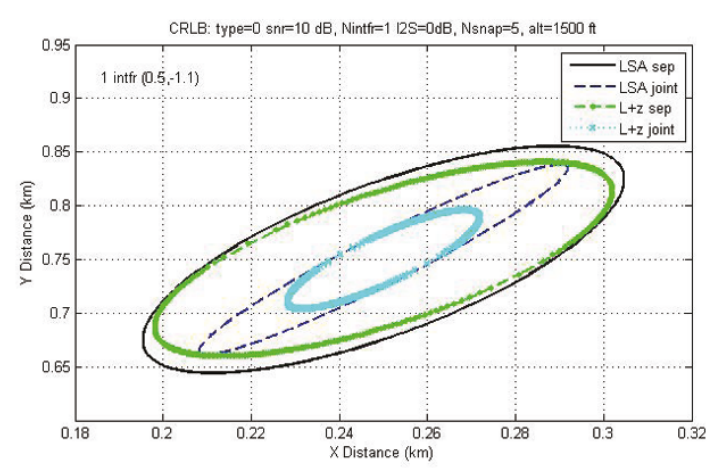

Figure $\mathbf{6} L+z$ array. (a) LSA vs. $L+z$ with no interference. (b) LSA vs. $L+z$ with one interferer.

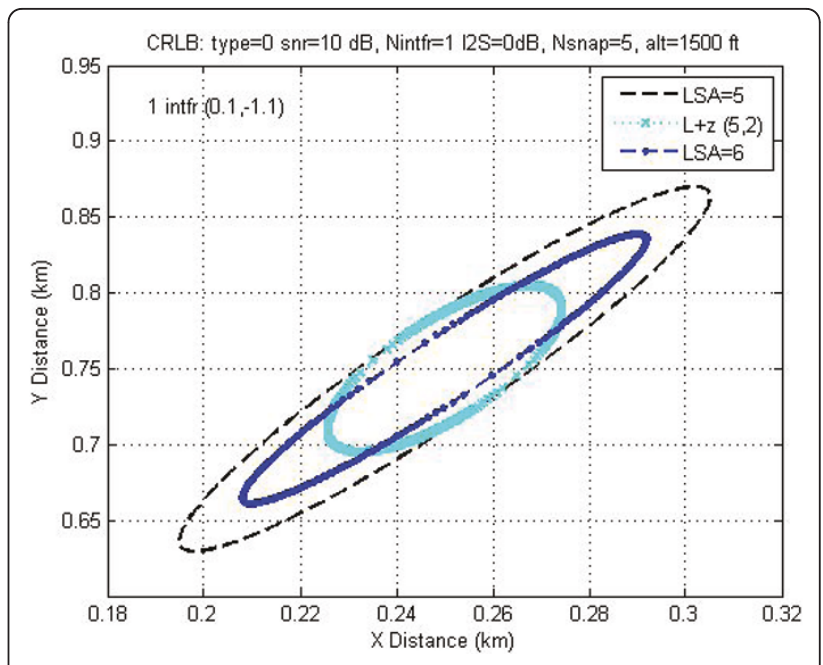

Figure $7 L+z$ with constant number of elements

In Figure 7 we repeat the comparison of LSA vs. $L+$ $z$, but with constant number of elements, and a single interferer. Take LSA, joint processing with $L=5$ (9 elements in total) as a baseline. Consider adding two additional elements, either the LSA with $L=6$, or the $L+z$ with $L=5$ and $z=2$, each having 11 elements in total. As seen, for this geometry, the $L+z$ is much preferred; the $L+z$ ellipse has the same width as the $L=5$ baseline, but much reduced length.

\section{Conclusions}

Two-dimensional angle-of-arrival estimation can be used to locate ground sources on the ground plane from a single array. The 2-D CRLB must be used to bound the performance, and we were able to find the needed bounds in the literature for both the Conditional Model Assumption (CMA) and the Unconditional Model Assumption (UMA). Some location systems may use suboptimal "separate" estimation of the two spatial angles, and this required us to modify the bounds. Our numerical examples show that joint processing has considerable advantage when interferers are present, particularly when an interferer lies near the "ambiguity cone" of the source. We also showed that adding additional elements along the $z$-axis can be very beneficial for distant sources when joint processing is used.

\section{Endnotes}

${ }^{a}[10,11]$ give the second term inside the braces as $\mathbf{P}^{\mathrm{T}} \otimes \mathbf{1}_{2} \mathbf{1}_{2}^{\mathrm{T}}$ and give $\mathbf{D}$ in a "parameters-first" ordering. Elementary permutation matrices can be used to reorder their D to be consistent with (3.9) in "users-first" ordering, which changes (3.8) to the form shown. Users-first ordering is preferred to analyze the "separate" bounds. 


\section{Abbreviations}

AOA: Angle-of-arrival; CMA: Conditional Model Assumption; CRLB: CramerRao lower bound; CF: confidence factor; LSA: L-shaped array; 1-D: onedimensional; psd: positive semi-definite; RF: radio frequency; 2-D: twodimensional; 2LSA: two L-shaped array; UXA: Uniform Cross Array; UMA: Unconditional Model Assumption.

\section{Competing interests}

The author declares that they have no competing interests.

Received: 30 November 2010 Accepted: 13 May 2011

Published: 13 May 2011

\section{References}

1. H Krim, M Viberg, Two Decades of Array Signal Processing Research. IEEE Signal Processing Magazine. (1996)

2. HL van Trees, Optimum Array Processing: Detection, Estimation, and Modulation Theory, Part IV. (Wiley-Interscience, Hoboken, NJ, 2002)

3. P Stoica, A Nehorai, MUSIC, Maximum-Likelihood, and Cramer-Rao bound IEEE Transactions on Acoustics, Speech, and Signal Processing. 37(5) (1989)

4. P Stoica, A Nehorai, Performance study of conditional and unconditional direction-of-arrival estimation. IEEE Transactions on Acoustics, Speech, and Signal Processing. 38(10) (1990)

5. P Stoica, E Larsson, A Gershman, The Stochastic CRB for array processing: a textbook derivation. IEEE Signal Processing Letters. 8(5) (2001)

6. N Tayem, H Kwon, L-shape 2-dimensional arrival angle estimation with propagator method. IEEE Transactions in Antennas and Propagation. 53(5) (2005)

7. S Kikuchi, H Tsuji, A Sano, Pair-matching method for estimating 2-D angle of arrival with a cross-correlation matrix. IEEE Antennas and Wireless Propagation Letters. 5 (2006)

8. T Xia, Y Zheng, Q Wan, X Wang, Decoupled estimation of 2-D angles of arrival using two parallel uniform linear arrays. IEEE Transactions on Antennas and Propagation. 55(9) (2007)

9. M Li, L Gan, P Wei, Improvement of 2-D direction finding algorithm based on two L-shape arrays. ICSP. (2008)

10. SZ Yau, Y Bresler, A compact Cramer-Rao bound expression for parametric estimation of superimposed signals. IEEE Transactions on Signal Processing 40(5) (1992)

11. ST Smith, Statistical resolution limits and the complexified Cramer-Rao bound. IEEE Transactions on Signal Processing. 53(5) (2005)

12. A Nehorai, E Paldi, Vector-sensor array processing for electromagnetic source localization. IEEE Transactions on Signal Processing. 42(2) (1994)

13. DT Vu, A Renaux, R Boyer, S Marcos, Performance analysis of $2 \mathrm{D}$ and $3 \mathrm{D}$ antenna arrays for source localization. 18th European Signal Processing Conference, EUSIPCO-2010

14. G Strang, Linear Algebra and its Applications. (Harcourt, Brace, Jovanovich, San Diego, CA, 1988)

15. LL Scharf, Statistical Signal Processing. (Addison-Wesley Publishing, New York, 1991)

16. HL van Trees, Detection, Estimation, and Modulation Theory: Part I. (Wiley, New York, 1968)

17. Y Hua, T Sarkar, D Weiner, An L-shaped array for estimating 2-D directions of wave arrival. IEEE Transactions on Antennas and Propagation. 39(2) (1991)

18. T Kailath, Linear Systems. (Prentice-Hall Inc, Englewood Cliffs, NJ, 1980)

doi:10.1186/1687-6180-2011-5

Cite this article as: Mailaender: Bounds for 2-D angle-of-arrival estimation with separate and joint processing. EURASIP Journal on Advances in Signal Processing 2011 2011:5

\section{Submit your manuscript to a SpringerOpen ${ }^{\circ}$ journal and benefit from:}

- Convenient online submission

- Rigorous peer review

- Immediate publication on acceptance

- Open access: articles freely available online

- High visibility within the field

- Retaining the copyright to your article

Submit your next manuscript at $>$ springeropen.com 